\title{
EDUCAÇÃO EM SAÚDE: UMA FERRAMENTA PARA A PREVENÇÃO E
}

\section{CONTROLE DE PARASITOSES}

\author{
Denize Ferreira RIBEIRO ${ }^{1}$ \\ Bianca Rafaela CORREIA ${ }^{2}$ \\ Adélia Karla Falcão SOARES ${ }^{3}$ \\ Mirella Katiane Leite da ROCHA ${ }^{4}$ \\ Estela Rodrigues Paiva ALVES \\ Mônica Camelo Pessoa de Azevedo ALBUQUERQUE ${ }^{6}$
}

${ }^{1}$ Graduanda de Enfermagem da Universidade Federal de Pernambuco (UFPE), Brasil. Discente integrante do grupo PET- Parasitologia da UFPE. Email: denizeg3@gmail.com

${ }^{2}$ Graduanda de Enfermagem da Universidade Federal de Pernambuco (UFPE), Brasil. Discente integrante do grupo PET- Parasitologia da UFPE. E-mail: bianca.rafaela.c@gmail.com

${ }^{3}$ Graduanda de Enfermagem da Universidade Federal de Pernambuco (UFPE), Brasil. Discente integrante do grupo PET- Parasitologia da UFPE. Email: adeliakarla@ hotmail.com

${ }^{4}$ Graduanda de Enfermagem da Universidade Federal de Pernambuco (UFPE), Brasil. Discente integrante do grupo PET- Parasitologia da UFPE. E-mail: mirellakatiane@gmail.com

${ }^{5}$ Mestre em Enfermagem em Promoção à Saúde pelo Programa Associado de Pós-Graduação da Universidade de Pernambuco e Universidade Estadual da Paraíba (PAPGEn-UPE/UEPB). Professora substituta da Universidade Federal de Pernambuco (UFPE). Brasil. E-mail:rodrigues.estela@gmail.com

${ }^{6}$ Doutora em Ciências Biológicas pela Universidade Federal de Pernambuco (UFPE). Professora Associada da Disciplina de Parasitologia do Departamento de Medicina Tropical da UFPE e Tutora do grupo PET PARASITOLOGIA (MEC-SESU). Pesquisadora do Laboratório de Imunopatologia Keizo Asami-LIKA (UFPE). Recife (PE), Brasil. E-mail: jcmonica@globo.com

\section{Recebido em: 29/10/2013 - Aprovado em: 21/12/2013 - Disponibilizado em: 15/01/2014}

RESUMO: A educação em saúde é uma prática que estimula mudanças de hábitos, melhoria da qualidade de vida e diminuição da morbimortalidade. O presente trabalho trata-se de uma revisão integrativa com o objetivo de avaliar a educação em saúde como instrumento eficiente no controle e prevenção de parasitoses. Para nortear este estudo utilizamos o seguinte questionamento: A educação em saúde tem sido uma ferramenta eficiente no controle e prevenção de doenças parasitárias? Foi realizada uma seleção de artigos utilizando os Descritores em Ciências da Saúde (DeCS): Educação em saúde, Doenças parasitárias, Parasitic diseases e Health education junto àbase de dados Lilacs, Medline e Scielo. Foram identificados nove artigos que atenderam a proposta do estudo e os resultados encontrados apontam para eficacia da educação em saúde na prevenção de parasitoses, devendo essa atividade ser contínua. Portanto, a educação em saúde é uma importante aliada quando se visa romper com modelos hegemônicos e convencionais de saúde que já demonstraram ser apenas paliativos.

Palavras-chave: Educação em saúde. Doenças parasitárias. Prevenção e controle. Promoção da saúde. Prevenção de doenças.

ABSTRACT: Health education is a practice that encourages changing habits, improving quality of life and decreased morbidity. The present work is an integrative review with the aim of evaluating health education as an efficient instrument in the control and prevention of parasitic diseases. To guide this study, we used the following question: Health education has been an effective tool in the prevention and control of parasitic diseases? We performed a selection of articles using the Descriptors in Health Sciences (DeCS): Educaçãoemsaúde, Doençasparasitárias, Parasitic diseases e Health education at the base of Lilacs, Medline and Scielo. Nine articles were identified that met the study proposal and the results indicate effectiveness of health education in the prevention of parasitic infections, such activity 
should be continuous. Therefore, health education is an important ally when it aims to break with the hegemonic models and conventional health that have shown to be only palliative.

Key-words: Health education. Parasitic Diseases.Prevention and control.Health promotion.Preventionofdiseases.

\section{Introdução}

As doenças parasitárias, cujos agentes etiológicos podem ser helmintos e protozoários constituem-se uma das maiores causas de morbidade e mortalidade em muitos países localizados nos trópicos, sendo endêmicas nos países em desenvolvimento. Segundo dados da Organização Mundial de Saúde, essas doenças afetam bilhões de pessoas levando a óbito, anualmente, outras milhões. É estimado que uma pessoa em cada quatro encontra-se infectada (WORLD HEALTH ORGANIZATION, 2013).

A malária representa a mais importante das doenças parasitárias com cerca de 219 milhões de casos e uma estimativa de 660.000 mortes (WORLD HEALTH ORGANIZATION, 2013). Em seguida está a esquistossomose cuja estimativa é de 200 milhões de pessoas infectadas em todo mundo e cerca de 20.000 mortes associadas às graves consequências da doença, e em terceiro lugar a amebíase, presente em todos os países tropicais em desenvolvimento, atingindo uma prevalência de $50 \%$ da população geral e causando aproximadamente 100.000 mortes por ano. Podemos citar, ainda, a leishmaniose, com prevalência mundial de aproximadamente 12 milhões de casos e mortalidade anual de cerca de 600.000 e a ancilostomose, que atinge cerca de 740 milhões de pessoas em países em desenvolvimento localizados nos trópicos (WORLD HEALTH ORGANIZATION, 2013b).

Apesar de possuir uma alta prevalência a nível mundial, essas doenças podem ser prevenidas ou até mesmo erradicadas com a melhoria do acesso a água potável e intervenções integradas como a educação em saúde e medidas sanitárias (WORLD HEALTH ORGANIZATION, 2006).

Apesar da existência de incentivo a pesquisas relacionadas às doenças parasitárias, a produção científica resultante dessas pouco contribui para a melhoria no arsenal terapêutico, educação em saúde e novos métodos de diagnóstico, essenciais ao combate dessas doenças (BRASIL, 2010).

Um dos principais fatores que contribuem para a elevada prevalência, assim como o surgimento de novas infecções parasitárias são as precárias condições de vida e saneamento básico deficiente ou mesmo inexistente, associada à falta de conhecimento da população sobre a transmissão e controle dessas infecções e princípios de higiene pessoal e cuidados no preparo correto dos 
alimentos. Assim, a implantação de práticas educativas que conduzam as pessoas a adquirir conhecimentos sobre as parasitoses, tornando-as capazes de atuarem na prevenção e redução da carga parasitária é uma estratégia eficaz, mas pouco trabalhada, pois o sistema não privilegia a educação em saúde, o que dificulta a implantação das ações de controle destas doenças (BOIA, 2006).

O princípio de se educar para saúde e para o ambiente, parte da hipótese de que vários problemas de saúde são resultantes da precária situação educacional da população, carecendo, portanto, de medidas "corretivas" e/ou educativas (ANDRADE et al., 2010).

A Educação em Saúde requer metodologias e teorias que permitam a construção do conhecimento compartilhado de forma contínua (EBLING et al., 2012). É imprescindível conhecer a realidade dos indivíduos com as quais se deseja programar uma ação educativa, conhecendo o seu contexto sociocultural e identificando as desigualdades sociais geradas pela pobreza (UCHÔA et al., 2009). Portanto, o controle e prevenção das doenças parasitárias são possíveis por meio de medidas simples que levem às pessoas a melhorarem sua qualidade de vida. A educação em saúde é uma ferramenta importante que ainda requer investimentos por partes dos líderes governamentais, vontade política, capacitação e incentivo dos profissionais que atuam no sistema público de saúde (HOTEZ et al., 2009).

\section{Metodologia}

Trata-se de uma revisão integrativa da literatura sobre o emprego da educação em saúde como uma ferramenta eficiente no controle e prevenção de doenças parasitárias.

Para nortear este estudo utilizamos o seguinte questionamento: $\mathrm{O}$ emprego da educação em saúde tem sido uma ferramenta eficiente no controle e prevenção de doenças parasitárias?

Foram consultadas as bases de dados

Lilacs (Literatura Latino-Americana e do Caribe em Ciências da Saúde), MedLine (Medical Literature Analysis and Retrieval System Online) e Scielo (Scientific Electronic Library Online) por intermédio da pesquisa online a partir do portal Biblioteca Virtual em Saúde (BVS). A busca foi realizada no período compreendido de 2002 a 2013.

Os artigos obedeceram aos seguintes critérios de inclusão: artigos publicados em português, inglês ou espanhol com resumos disponíveis nas bases de dados selecionadas; artigos disponíveis na íntegra no portal da BVS, período de publicação de 2002 a 2013 e artigos que abordassem a temática educação em saúde no controle e prevenção das doenças parasitárias. Como critério de exclusão: artigos publicados nos demais idiomas, anteriores ao ano de 2002, artigos não disponíveis na íntegra online e que não respondessem a pergunta norteadora, ou seja, que não avaliassem a eficácia de um processo educativo. 
As palavras-chave utilizadas foram: doenças parasitárias, educação em saúde, health education e parasitic diseases. $\mathrm{O}$ cruzamento dos descritores foi feito utilizando o operador booleano “AND”: Doenças parasitárias “AND” educação em saúde; Parasitic diseases "AND” health education.

Os dados foram coletados por quatro pesquisadores e os resultados obtidos foram apresentados de forma descritiva. Em uma primeira etapa, eles foram sintetizados e organizados num documento contendo informações como título da pesquisa, nome dos autores, ano, objetivo, delineamento metodológico, síntese, resultados e níveis de evidência, servindo como um banco de dados de fácil manejo das informações contidas em cada artigo. Na segunda etapa, os dados foram organizados e sintetizados conforme os seguintes parâmetros: título, autoria/ano, objetivos e principais resultados.

\section{Resultados}

Por meio da pesquisa feita junto às bases de dados virtual descritas anteriormente, foram localizados 635 estudos, assim distribuídos nas bases de dados: MEDLINE-449, LILACS-159 e Scielo-27. Destes, apenas nove artigos atenderam a proposta do estudo.

Em relação ao ano de publicação dos estudos analisados, um ocorreu em 2005, quatro em 2009, três em 2011 e um em 2012.

Os países nos quais ocorreram os estudos foram assim dispostos: Brasil com seis artigos e Cuba, Camarões e Argentina com um artigo cada.

Os artigos dos estudos foram assim estão distribuídos nos seguintes periódicos: Revista da Sociedade Brasileira de Medicina Tropical, Revista Brasileira de Promoção da Saúde, Revista RENE, Revista de Saúde Pública, Revista Panamericana de Salud Pública, Online Brazil Journal Nursing, Revista Archivo de Camaguey, Pan African Medical Journal, e Parasitology.

No que concerne ao tipo de pesquisa, foram encontrados um estudo pesquisa-ação (Pereira et al. 2012), um estudo experimental (Kamga et al. 2011), um relato de experiência (Joventino et al. 2009), um estudo longitudinal (Toledo et al. 2009), dois estudos descritivos (Barbosa et al. 2009; Joventino et al. 2011) e três estudos do tipo quase-experimental (Pezzani et al. 2009; Rodríguez et al. 2011; Ferreira e Andrade 2005).

Os artigos foram sintetizados e sumarizados no quadro 1 de acordo com: Autoria/Ano, Objetivos/Métodos, Resultados/Discussão e Conclusões/Recomendações. 
Quadro 1- Publicações relativas à temática Educação em saúde na prevenção e controle das doenças parasitárias

\begin{tabular}{|c|c|c|c|}
\hline Autor/Ano & Objetivos/Métodos & Resultados/Discussão & Conclusões/ Recomendações \\
\hline $\begin{array}{l}\text { Joventino ES } \\
\text { et al. } \\
2009\end{array}$ & 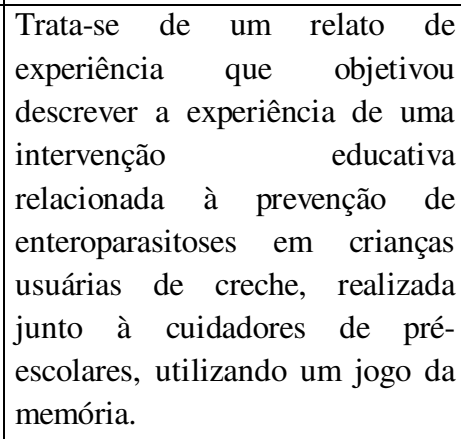 & $\begin{array}{l}\text { Todos participaram ativamente na } \\
\text { construção do conhecimento. A ação } \\
\text { contribuiu para ampliar o } \\
\text { conhecimento dos participantes sobre } \\
\text { a importância da prevenção de } \\
\text { enteroparasitoses e incorporação dos } \\
\text { conceitos à sua realidade. O aumento } \\
\text { da porcentagem de acertos avaliados } \\
\text { ratificou a eficácia da atividade } \\
\text { educativa. }\end{array}$ & $\begin{array}{l}\text { A estratégia educativa possibilitou } \\
\text { uma melhor compreensão sobre a } \\
\text { prevenção das enteroparasitoses } \\
\text { por parte dos cuidadores, sendo } \\
\text { bastante eficiente. }\end{array}$ \\
\hline $\begin{array}{l}\text { Barbosa LA } \\
\text { et al. }\end{array}$ & $\begin{array}{l}\text { Estudo descritivo cujo objetivo } \\
\text { foi avaliar a sensibilização de } \\
\text { crianças e seus responsáveis } \\
\text { sobre prevenção de parasitoses } \\
\text { na infância, usando o lúdico para } \\
\text { crianças e uma roda de conversa } \\
\text { para os responsáveis. }\end{array}$ & $\begin{array}{l}\text { Tanto as crianças como seus } \\
\text { responsáveis demonstraram ter } \\
\text { compreendido a } \\
\text { educativa, participando ativamente } \\
\text { das atividades. As estratégias de } \\
\text { abordagem participativa mostraram } \\
\text { ser adequadas para o } \\
\text { desenvolvimento de uma proposta } \\
\text { educativa eficaz. }\end{array}$ & 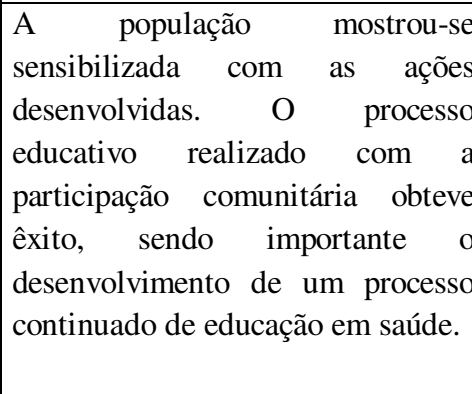 \\
\hline $\begin{array}{l}\text { Toledo MJO } \\
\text { et al. } \\
2009\end{array}$ & $\begin{array}{l}\text { Estudo longitudinal que teve } \\
\text { como objetivo analisar o estado } \\
\text { de infecção enteroparasitária em } \\
\text { famílias de comunidade indígena } \\
\text { após instituição de medidas de } \\
\text { controle para enteroparasitos, } \\
\text { utilizando a elaboração de um } \\
\text { caderno bilíngue de apoio. }\end{array}$ & $\begin{array}{l}\text { A prevalência de algumas espécies de } \\
\text { parasitos reduziu, enquanto que a de } \\
\text { outras aumentou significativamente, } \\
\text { embora tenham sido observadas } \\
\text { variações importantes nas condições } \\
\text { de moradia e higiene após a ação } \\
\text { desenvolvida. }\end{array}$ & $\begin{array}{l}\text { Apesar do aumento da prevalência } \\
\text { de algumas espécies de parasitos, } \\
\text { houve um impacto positivo nos } \\
\text { indicadores parasitológicos de } \\
\text { saúde, por isso, a importância das } \\
\text { atividades de educação em saúde } \\
\text { continuadas e a manutenção das } \\
\text { melhorias sanitárias. }\end{array}$ \\
\hline $\begin{array}{l}\text { Pezzani BC } \\
\text { et al. } \\
2009\end{array}$ & $\begin{array}{l}\text { Trata-se de um estudo quase- } \\
\text { experimental com objetivo de } \\
\text { avaliar um plano de ações para } \\
\text { redução de parasitoses, contando } \\
\text { com realização de exames } \\
\text { coproparasitológicos } \\
\text { intervenção educativa. Foram } \\
\text { realizados reuniões educativas } \\
\text { com a comunidade abordando } \\
\text { medidas preventivas. }\end{array}$ & $\begin{array}{l}\text { A quimioterapia antiparasitária foi } \\
\text { eficaz em mais de } 85 \% \text { para todos os } \\
\text { parasitos identificados. Observou-se } \\
\text { uma mudança dos hábitos de higiene. } \\
\text { O tratamento dos indivíduos } \\
\text { infectados e a intervenção educativa } \\
\text { envolvendo promotores locais } \\
\text { permitiram reduzir as doenças } \\
\text { parasitárias, sobretudo à prevalência } \\
\text { dos helmintos. }\end{array}$ & $\begin{array}{l}\text { O estudo mostrou que o plano de } \\
\text { ações reduziu as taxas de infecções } \\
\text { parasitárias, principalmente as } \\
\text { infecções por helmintos. }\end{array}$ \\
\hline $\begin{array}{l}\text { Joventino ES } \\
\text { et al. } \\
2011\end{array}$ & $\begin{array}{l}\text { Estudo descritivo cujo objetivo } \\
\text { foi avaliar o processo de } \\
\text { mudanças comportamentais dos } \\
\text { cuidadores de crianças em idade } \\
\text { pré-escolar, em relação à } \\
\text { prevenção de parasitoses } \\
\text { intestinais, com a aplicação de } \\
\text { um jogo da memória e discussões } \\
\text { em grupo. }\end{array}$ & $\begin{array}{l}\text { Mesmo com a ação educativa e com o } \\
\text { reforço oferecido de informações } \\
\text { relacionadas à prevenção de } \\
\text { enteroparasitoses, o comportamento } \\
\text { dos cuidadores nem sempre foi } \\
\text { considerado adequado, sendo } \\
\text { observado que as medidas profiláticas } \\
\text { não eram colocadas em prática } \\
\text { devido às condições de vida precárias } \\
\text { enfrentadas pelos participantes. }\end{array}$ & $\begin{array}{l}\text { Um acompanhamento efetivo é } \\
\text { necessário para despertar nas } \\
\text { famílias a urgência da adoção de } \\
\text { medidas profiláticas. }\end{array}$ \\
\hline
\end{tabular}


Quadro 1, continuação

\begin{tabular}{|c|c|c|c|}
\hline $\begin{array}{l}\text { Kamga HLF } \\
\text { et al. } \\
2011\end{array}$ & 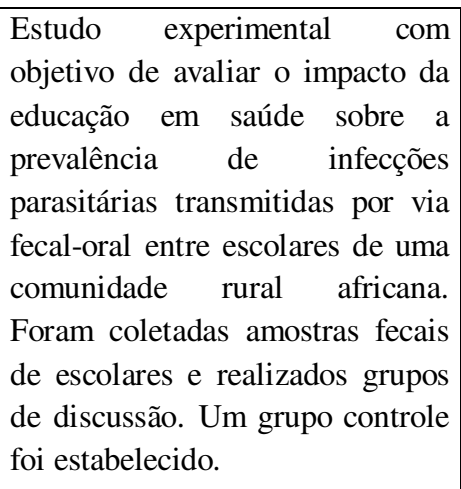 & $\begin{array}{l}\text { Houve uma queda significativa na } \\
\text { prevalência dos parasitos na aldeia } \\
\text { onde a educação em saúde foi } \\
\text { realizada. Na aldeia na qual nenhuma } \\
\text { ação de educação em saúde foi feita a } \\
\text { redução não foi significativa. Não } \\
\text { houve diferença na prevalência } \\
\text { quando comparada as diferenças de } \\
\text { idade entre os escolares. }\end{array}$ & $\begin{array}{l}\text { A aldeia que recebeu a intervenção } \\
\text { em saúde teve a prevalência de } \\
\text { infecções parasitárias diminuída } \\
\text { significativamente, demonstrando } \\
\text { a importância das ações educativas } \\
\text { desenvolvidas. }\end{array}$ \\
\hline $\begin{array}{l}\text { Rodríguez JE } \\
\text { et al. } \\
2011\end{array}$ & $\begin{array}{l}\text { Estudo quase-experimental que } \\
\text { teve como objetivo promover o } \\
\text { aumento do nível de } \\
\text { conhecimento sobre as medidas } \\
\text { de controle de parasitoses por } \\
\text { meio de uma intervenção } \\
\text { educativa aplicada a mães em um } \\
\text { consultório pediátrico, utilizando } \\
\text { um material de estudo entregue } \\
\text { as mesmas, sendo avaliados os } \\
\text { conhecimentos adquiridos pós- } \\
\text { leitura. }\end{array}$ & $\begin{array}{l}\text { Depois da intervenção, } 100 \% \text { das } \\
\text { mães reconheceram como medidas } \\
\text { preventivas necessárias para o } \\
\text { controle das parasitoses: a lavagem } \\
\text { das mãos, frutas e vegetais, fervura } \\
\text { ou cloração da água, evitar o } \\
\text { transbordamento de esgotos e } \\
\text { proteger alimentos. }\end{array}$ & $\begin{array}{l}\text { A intervenção foi considerada } \\
\text { satisfatória, observando que houve } \\
\text { um aumento do conhecimento dos } \\
\text { temas abordados. }\end{array}$ \\
\hline $\begin{array}{l}\text { Ferreira GR e } \\
\text { Andrade CFS } \\
2005\end{array}$ & $\begin{array}{l}\text { Estudo quase-experimental com } \\
\text { objetivo de avaliar a prevalência } \\
\text { de parasitos intestinais, aspectos } \\
\text { epidemiológicos e socioculturais } \\
\text { e avaliar uma intervenção } \\
\text { educativa sobre parasitoses } \\
\text { intestinais entre os escolares. } \\
\text { Foram coletadas amostras fecais } \\
\text { para exames parasitológicos, } \\
\text { realizados questionários e uma } \\
\text { oficina de trabalho para os pais e } \\
\text { alunos. }\end{array}$ & $\begin{array}{l}\text { Foi observada uma prevalência de } \\
11,5 \% \text { para pelo menos um parasito } \\
\text { intestinal. Dos } 200 \text { participantes da } \\
\text { oficina de trabalho, } 75 \% \text { (150 } \\
\text { escolares e pais/responsáveis) } \\
\text { responderam ao questionário de } \\
\text { conhecimentos sobre as parasitoses, } \\
\text { onde foram obtidos resultados } \\
\text { significativos, notando-se que houve } \\
\text { ampla assimilação dos conhecimentos } \\
\text { e mudanças nos hábitos e conceitos } \\
\text { apontados anteriormente. }\end{array}$ & $\begin{array}{l}\text { As práticas educacionais quando } \\
\text { bem aplicadas levam as pessoas a } \\
\text { adquirirem os conhecimentos para } \\
\text { prevenção de parasitoses, } \\
\text { alcançando objetivos propostos. } \\
\text { Apesar de importantes, as } \\
\text { atividades educativas precisam ser } \\
\text { integradas a um processo contínuo } \\
\text { de educação e controle das } \\
\text { enteroparasitoses. }\end{array}$ \\
\hline $\begin{array}{l}\text { Pereira AP } \\
\text { et al. } \\
2012\end{array}$ & $\begin{array}{l}\text { Trata-se de um estudo pesquisa- } \\
\text { ação objetivando avaliar a } \\
\text { influência de uma intervenção } \\
\text { educativa com leituras, debates e } \\
\text { workshops na prevalência de } \\
\text { infecções parasitárias intestinais } \\
\text { por meio de um questionário pré } \\
\text { e pós-teste e exames } \\
\text { coproparasitológicos. }\end{array}$ & $\begin{array}{l}\text { A população do estudo apresentou } \\
\text { maior consciência da importância do } \\
\text { parasitismo intestinal, evidenciado } \\
\text { nas mudanças de atitudes e práticas } \\
\text { relacionadas à prevenção observadas } \\
\text { antes e depois das intervenções } \\
\text { educativas. }\end{array}$ & $\begin{array}{l}\text { O estudo permitiu a construção } \\
\text { compartilhada de conhecimento, } \\
\text { que refletiu as reais necessidades } \\
\text { dos moradores. A promoção da } \\
\text { saúde exige a participação popular, } \\
\text { o envolvimento da comunidade } \\
\text { científica e envolvimento das } \\
\text { autoridades. }\end{array}$ \\
\hline
\end{tabular}




\section{Discussão}

De acordo com os resultados obtidos nas referidas bases, verifica-se um relativo aumento das produções nos anos de 2009 e 2011, com uma diminuição no ano subsequente. Nota-se, também, que seis publicações $(66,6 \%)$ foram a nível nacional e três $\quad(33,4 \%) \quad$ publicações foram internacionais, ressaltando a maior importância dada ao tema educação em saúde aplicada às doenças parasitárias a nível nacional.

Foram observadas variadas intervenções envolvendo a população de estudo em diversos cenários, tais como: escolas, hospitais e em domicílio, enfatizando medidas profiláticas e ações de controle, bem como o conhecimento geral da doença como formas de transmissão, tratamento e ciclo biológico do parasito.

Alguns estudos realizaram intervenções direcionadas aos profissionais de saúde e professores do ensino fundamental (TOLEDO et al., 2009; JOVENTINO et al., 2009; JOVENTINO et al., 2011). O envolvimento desses profissionais, como forma de atualização e treinamento para abordar o tema posteriormente, é uma estratégia decisiva para multiplicação da ação educativa.

Também foram identificados estudos com intervenções educativas direcionadas a crianças em idade escolar (FERREIRA e
ANDRADE, 2005; BARBOSA et al., 2008;

TOLEDO et al., 2009; KAMGA et al., 2011). As crianças geralmente são mais vulneráveis a adquirir doenças por via fecal-oral e nestes indivíduos as infecções por parasitos intestinais assumem grande importância, não só pela morbidade resultante, mas também pela frequência com que produzem déficit que pode comprometer o desenvolvimento físico e cognitivo, resultando até mesmo em óbito. Além disso, o ambiente escolar proporciona uma grande circulação e transmissão desses agentes infecciosos devido ao aumento do contato interpessoal, maior contato com o solo e precárias condições de higiene inerentes à exploração da fase oral pelas crianças (BARÇANTE et al., 2008). Barbosa et al. (2008) comentam, ainda, que ao se trabalhar a educação em saúde na infância aumentam-se as chances de que estas alcancem na idade adulta uma maior qualidade de vida, consciência crítica e autonomia.

Sabe-se que os aspectos sociais e ambientais estão envolvidos na problemática das parasitoses, tornando-se de extrema importância o conhecimento desses aspectos quando se visa realizar uma intervenção educativa de maneira eficaz. Todos os artigos analisados se preocuparam com a análise dos aspectos socioeconômicos e ambientais. Isto é importante, pois auxilia no desenvolvimento de parâmetros que irão conduzir às ações educativas, criando uma estratégia de ensino adequada às peculiaridades da comunidade. 


\section{A aproximação da intervenção} educativa de métodos e técnicas pedagógicas, em oposição aos modelos tradicionais de ensino em que a informação é simplesmente fornecida ao indivíduo, pode potencializar a ação em saúde, favorecendo a maior participação dos sujeitos envolvidos, o debate e a autonomia, além de garantir o exercício da cidadania. Foi observado que em todos os artigos foi utilizado um método de ensino participativo, adequando-se a faixa etária do público alvo.

A falta de uma estratégia diversificada em educação dificulta o entendimento dos participantes de um determinado estudo, quando estes não contam com um material que facilite a compreensão e a mudança dos hábitos de forma eficaz (MAZZIONI, 2009). Todos os trabalhos analisados fizeram uso de materiais didáticos de apoio, por exemplo: jogos educacionais, fantoches, recursos áudiovisuais, cartazes, livros e cartas pictóricas.

O tempo foi apontado como um fator importante a ser considerado para a prática educativa em saúde. A educação deve-se estender por toda a vida, não se limitando a etapas ou períodos, visto que o homem é um ser em contínuo processo de mudanças e transformações (RODRIGUES, 2001). Barbosa et al. (2009); Toledo et a. (2009) e Ferreira et al. (2005) salientaram a importância de uma educação contínua e ampliada. Toledo et al. (2009) observaram em seu estudo que o tempo para realização das atividades educativas foi insuficiente, razão pela qual o número de indivíduos parasitados permaneceu elevado mesmo após a implantação de medidas de controle.

Em relação ao impacto alcançado, observou-se que as intervenções educativas foram decisivas para o aumento de conhecimento da população de cada estudo em questão. Em seus estudos, Kamga et al. (2011); Ferreira e Andrade (2005) e Andrade et al. (2010) trabalharam usando grupos experimentais e controles, sendo o grupo experimental marcado por receber intervenção educativa em saúde, o que demonstrou através de diferenças antes e após a intervenção, a importância do emprego da educação em saúde.

Apesar de ser observado um aumento no conhecimento sobre hábitos preventivos, a situação socioeconômica dos indivíduos participantes dos estudos ainda é relatada como um fator limitante da adoção de comportamentos e práticas preventivas de doenças e de promoção geral da saúde (BARBOSA, et al., 2008; JOVENTINO, et al., 2011). Portanto, os profissionais de saúde, enquanto principais disseminadores de mensagens educativas precisam despender esforços na execução de atividades educativas criativas que explorem meios de contornar as limitações propiciadas pela pobreza.

Adicionalmente, há a necessidade de maior atenção e planejamento dos dirigentes para captação de recursos financeiros a fim de ampliar territorialmente as ações de controle das parasitoses (FERREIRA e ANDRADE, 
2005). Nesse sentido, é importante que se busque ativamente parceria com autoridades a níveis distritais, nacionais e internacionais a fim de que uma estratégia desejada tenha um maior alcance e efetividade, uma vez que a promoção da saúde envolve a responsabilidade de vários atores da sociedade: estado, comunidade, família e indivíduo.

Alguns estudos tiveram apoio de organizações governamentais e não governamentais o que garantiu uma maior visibilidade ao programa de educação em saúde, cujo apoio foi extremamente importante para o sucesso de ações de prevenção e controle de doenças parasitárias.

O trabalho de Pereira et al. (2012) teve o suporte financeiro da Fundação Oswaldo Cruz (FIOCRUZ), Conselho Nacional de Desenvolvimento Científico e Tecnológico (MCT-CNPq) e da Fundação Carlos Chagas Filho de Amparo à Pesquisa do Estado do Rio de Janeiro (FAPERJ). Barbosa et al. (2009) realizou sua pesquisa em parceria com a equipe da Estratégia de Saúde da Família (ESF), tendo o apoio da Secretaria de Saúde Municipal de Crato - CE; e o projeto educativo de Pezzani et al. (2009) obteve o apoio da Agência Nacional para a Promoção da Ciência e Tecnologia da Fundação Alberto J. Roemmers e da Universidade Nacional de La Plata. O trabalho de Toledo et al. (2009) foi financiado pela Fundação Nacional de Saúde/Ministério da Saúde. Todos os estudos aqui descritos concordam com o fato de que a educação em saúde é extremamente importante e eficaz, mas que sua ação ganha força quando aliada a órgãos e programas de apoio governamentais.

\section{Conclusões}

A educação em saúde é uma ferramenta extremamente eficaz para melhoria da qualidade de vida das pessoas. É importante pensar nela como uma maneira de romper com modelos hegemônicos e convencionais de saúde que já demonstraram ser apenas paliativos.

São poucas as publicações relativas à temática Educação em Saúde no controle e prevenção de doenças parasitárias encontradas na literatura. Isto é justificado pelo maior enfoque dado aos aspectos individuais e curativos que se mostram superiores aos aspectos comunitários, preventivos e educativos necessários à sociedade.

Os estudos analisados revelaram que várias ferramentas educacionais podem ser utilizadas na ação de educar em saúde, com fortes evidências do efeito positivo do uso de estratégias inovadoras e participativas na ação educativa proposta.

Desta forma, a Educação em Saúde pode contribuir efetivamente para o controle $\mathrm{e}$ prevenção de parasitoses, podendo alcançar efeitos mais duradouros em comparação a outras abordagens, visto que inteirado de conhecimentos o indivíduo evita a aquisição das doenças parasitárias e de suas consequências. Além do mais, as ações 
exigem um custo menor, na maioria das vezes, quando comparado ao tratamento curativo, o que revela a sua importância na melhoria da qualidade de vida das pessoas. Por fim, é necessário que essas atividades não

\section{Referências Bibliográficas}

ANDRADE EC et al. Parasitoses intestinais: uma revisão sobre seus aspectos epidemiológicos, clínicos e terapêuticos. Rev. APS, Juiz de Fora. 2010; 13(2): 231 -240. http://www.aps.ufjf.br/index.php/aps/article/v iew/736/319

BARBOSA LA et al. A educação em saúde como instrumento na prevenção de parasitoses. Rev. Bras. Promoção da Saúde. RBPS. 2009; 22(4): 272 - 278. http://www.unifor.br/images/pdfs/rbps/artigo1 0 2009.4.pdf.

BARÇANTE TA et al. Enteroparasitoses em crianças matriculadas em creches públicas do município de Vespasiano, Minas Gerais. RPT. 2008; 37(1):33-42.

http://revista.iptsp.ufg.br/uploads/files/2008 3 7(1)33_42.pdf.

BÓIA MN et al. Mass treatment for intestinal helminthisis control in an Amazonian endemic area in Brazil. Rev. Inst. Med. Trop. S. Paulo. 2006; 48(4): 189-195. http://www.scielo.br/scielo.php?pid=S0036$\underline{46652006000400003 \& \text { script }=\text { sci_arttext }}$

BRASIL. Doenças negligenciadas: estratégias do Ministério da Saúde. Informe Técnico. Ministério da Saúde. Rev Saúde Pública. 2010; 44(1): 200-200 http://www.scielosp.org/pdf/rsp/v44n1/23.pdf

EBLING SBD et al. Popular education and health education: a necessary link in health practices. J Nurs UFPE on line. 2012; 6(9): 2285-9. sejam isoladas, sendo fundamental a participação popular, o envolvimento da comunidade científica e das autoridades nessas ações.

http://www.revista.ufpe.br/revistaenfermagem /index.php/revista/article/view/2584/pdf_1501

FERREIRA GR; ANDRADE CFS. Alguns aspectos socioeconômicos relacionados a parasitoses intestinais e avaliação de uma intervenção educativa em escolares de Estiva Gerbi, SP. Rev. Soc. Bras. Med. Trop. 2005; 38(5): 402-405. http://www.scielo.br/scielo.php?script=sci_art text\&pid=S0037-86822005000500008.

HOTEZ PJ et al. Rescuing the bottom billion through control of neglected tropical diseases. The Lancet. 2009; 373( 9674): 1570-1575. http://www.thelancet.com/journals/lancet/arti cle/PIIS0140-6736(09)60233-6/fulltext.

JOVENTINO ES et al. Jogo da memória como estratégia educativa para prevenção de enteroparasitoses: relato de experiência. Rev. Rene. 2009; 10( 2): 141-148. http://www.revistarene.ufc.br/vol10n2_pdf/a1 6v10n2.pdf.

JOVENTINO ES et al. Health education in the prevention of enteroparasites: descriptive study. Online Braz. J. Nurs. 2011; 10(2) http://www.objnursing.uff.br/index.php/nursi ng/article/view/3253/980.

KAMGA HLF et al. The impact of health education on the prevalence of faecal-orally transmitted parasitic infections among school children in a rural community in Cameroon. Pan Afr Med J. 2011; 8(38): 1-7. http://www.ncbi.nlm.nih.gov/pubmed/221214 46.

MAZZIONI S. As Estratégias Utilizadas No Processo De Ensino-Aprendizagem: 
Concepções De Alunos e Professores De Ciências Contábeis. In: Congresso USP de Controladoria e Contabilidade, Anais eletrônicos... São Paulo: USP, 2009. http://www.congressousp.fipecafi.org/artigos9 2009/283.

PEREIRA APMF et al. The influence of health education on the prevalence of intestinal parasites in a low-income community of Campos dos Goytacazes, Rio de Janeiro State, Brazil. Parasitology. 2012; 39(6): 791-801. http://www.ncbi.nlm.nih.gov/pubmed/223135 $\underline{20}$.

PEZZANI BC et al. A Participación comunitaria en el control de las parasitosis intestinales en una localidad rural de Argentina. Rev Panam Salud Publica. 2009; 26(6): 471- 477.

http://www.scielosp.org/scielo.php?script=sci _arttext\&pid=S1020-49892009001200001.

RODRIGUES N. Educação: da formação humana à construção do sujeito ético. Educ. Soc.2001; 22(76): 232-257. http://www.scielo.br/pdf/es/v22n76/a13v2276 .pdf.

RODRÍGUEZ JE et al. Estrategia educativa para la prevención del parasitismo en edades pediátricas. Arch. M.D. Camaguey. 2011; 15(1): 1-11.

http://scielo.sld.cu/scielo.php?script=sci artte xt\&pid=S1025-02552011000100012.

TOLEDO MJO et al. Avaliação de atividades de controle para enteroparasitos em uma aldeia Kaingáng do Paraná. Rev Saúde Pública. 2009; 43(6): 981-90. http://www.scielo.br/scielo.php?pid=S0034$\underline{89102009005000083 \& \text { script=sci_arttext. }}$.

UCHÔA CMA et al. Parasitismo intestinal em crianças e funcionários de creches comunitárias na cidade de Niterói-RJ, Brasil.

Rev. de Patologia Tropical. 2009; 38(4): $267-$ 278.

http://revista.iptsp.ufg.br/uploads/63/original_ 2009_38_4_267_278.pdf.
WORLD HEALTH ORGANIZATION.

Malaria [Internet]. 2013a. http://www.who.int/mediacentre/factsheets/fs 094/en/.

WORLD HEALTH ORGANIZATION.

Parasitic diseases [Internet]. 2013b.

http://www.who.int/vaccine_research/diseases

/soa_parasitic/en/index.html.

WORLD HEALTH ORGANIZATION.

Neglected tropical diseases, hidden successes, emerging opportunities[Internet]. 2006.

http://whqlibdoc.who.int/hq/2006/WHO_CDS _NTD_2006.2_eng.pdf 